\title{
Article
}

\section{Defected and Functionalized Germanene based Nanosensors under Sulfur Comprising Gas Exposure}

Tanveer Hussain, thanayut kaewmaraya, Sudip Chakraborty, Hakkim Vovusha, Vittaya Amornkitbamrung, and Rajeev Ahuja

ACS Sens., Just Accepted Manuscript • DOI: 10.1021/acssensors.8b00167 • Publication Date (Web): 27 Mar 2018

Downloaded from http://pubs.acs.org on April 8, 2018

\section{Just Accepted}

"Just Accepted" manuscripts have been peer-reviewed and accepted for publication. They are posted online prior to technical editing, formatting for publication and author proofing. The American Chemical Society provides "Just Accepted" as a service to the research community to expedite the dissemination of scientific material as soon as possible after acceptance. "Just Accepted" manuscripts appear in full in PDF format accompanied by an HTML abstract. "Just Accepted" manuscripts have been fully peer reviewed, but should not be considered the official version of record. They are citable by the Digital Object Identifier (DOI®). "Just Accepted" is an optional service offered to authors. Therefore, the "Just Accepted" Web site may not include all articles that will be published in the journal. After a manuscript is technically edited and formatted, it will be removed from the "Just Accepted" Web site and published as an ASAP article. Note that technical editing may introduce minor changes to the manuscript text and/or graphics which could affect content, and all legal disclaimers and ethical guidelines that apply to the journal pertain. ACS cannot be held responsible for errors or consequences arising from the use of information contained in these "Just Accepted" manuscripts. 


\section{Defected and Functionalized Germanene based Nanosensors under Sulfur Comprising Gas Exposure}

Tanveer Hussain, ${ }^{1}$ Thanayut Kaewmaraya, ${ }^{2,3}$ Sudip Chakraborty ${ }^{*}{ }^{4}$ Hakkim Vovusha ${ }^{4,6}$

Vittaya Amornkitbamrung, ${ }^{2,3}$ and Rajeev Ahuja ${ }^{4,5}$

${ }^{1}$ Centre for Theoretical and Computational Molecular Science, Australian Institute for Bioengineering and Nanotechnology, The University of Queensland, Brisbane, Qld 4072, Australia

${ }^{2}$ Integrated Nanotechnology Research Center, Department of Physics, Khon Kaen University, Khon Kaen, Thailand

${ }^{3}$ Nanotec-KKU Center of Excellence on Advanced Nanomaterials for Energy Production and Storage, Khon Kaen, Thailand

${ }^{4}$ Condensed Matter Theory Group, Department of Physics and Astronomy, Box 516, Uppsala University, S-75120 Uppsala, Sweden

${ }^{5}$ Applied Materials Physics, Department of Materials and Engineering, Royal Institute of Technology (KTH), S-100 44 Stockholm, Sweden

${ }^{6}$ King Abdullah University of Science and Technology (KAUST), Physical Science and Engineering Division (PSE), Thuwal 23955-6900, Saudi Arabia

* sudiphys@,gmail.com 


\title{
Key words:
}

Binding characteristics, Nano sheets, Substitution, Nanostructures, Nanosensors

\begin{abstract}
:
Efficient sensing of sulfur containing toxic gases like $\mathrm{H}_{2} \mathrm{~S}$ and $\mathrm{SO}_{2}$ is of outmost importance due to the adverse effects of these noxious gases. Absence of an efficient 2D based nanosensors capable of anchoring $\mathrm{H}_{2} \mathrm{~S}$ and $\mathrm{SO}_{2}$ with feasible binding and an apparent variation in electronic properties upon the exposure of gas molecules has motivated us to explore the promise of germanene nano sheet (Ge-NS) for this purpose. In the present study, we have performed a comprehensive computational investigation by means of DFT based first principles calculations to envisage the structural, electronic and gas sensing properties of pristine, defected and metal substituted Ge-NS. Our initial screening has revealed that although interaction of $\mathrm{SO}_{2}$ on pristine Ge-NS is within the desirable range, however $\mathrm{H}_{2} \mathrm{~S}$ binding is falling below the required values to guarantee an effective sensing. To improve the binding characteristics, we have considered the interactions between $\mathrm{H}_{2} \mathrm{~S}$ and $\mathrm{SO}_{2}$ with defected and metal substituted Ge-NS. The systematic removals of Ge atoms from a reasonably large super cell lead to mono-vacancy, di-vacancies and tri-vacancies in Ge-NS. Similarly, different transition metals like $\mathrm{As}, \mathrm{Co}, \mathrm{Cu}, \mathrm{Fe}, \mathrm{Ga}, \mathrm{Ge} \mathrm{Ni}$ and $\mathrm{Zn}$ have been substituted into the monolayer to realize substituted Ge-NS. Our van der Waals corrected DFT calculations have concluded that the vacancy and substitution defects not only improve the binding characteristics but also enhance the sensing propensity of both $\mathrm{H}_{2} \mathrm{~S}$ and $\mathrm{SO}_{2}$. The total and projected density of states show significant variations in electronic properties of pristine and defected Ge-NS before and after the exposure to the gases, which are essential in constituting a signal to be detected by the external circuit of the sensor. We strongly believe that out present work would not only advance the knowledge towards the application of GeNS based sensing, but also provide the motivation for the synthesis of an efficient nanosensors for $\mathrm{H}_{2} \mathrm{~S}$ and $\mathrm{SO}_{2}$.
\end{abstract}


The search for novel and less expensive sensing materials with high sensitivity and selectivity has been triggered in the scientific community recently ${ }^{1-3}$. High surface-to-volume ratio is always desirable for a suitable gas sensor, which enables nanostructures to be emerged as the most promising materials with large active surface area that can interact with gas molecules in the environment ${ }^{4}$. As a consequence of this large interaction area, one can expect enhancement in the sensitivity of the sensor devices based on such nanostructures ${ }^{5-14}$. In addition to the large surface area, high electrical conductivity and low noise of the twodimensional (2D) nano-structures advancing them ahead of one-dimensional (1D) structures like nanotubes or nanowires. Because of the lower electrical noise corresponding to a higher electrical conductivity, significant conductivity change could be occurred even when a gas molecule induces small carrier concentration. The charge transfers between 2D surface and the gas molecule also favour these materials best suited for gas sensor fabrication.

Graphene with its unique electronic properties and lower electrical noise shows high electron mobility and therefore being sensitive to the chemical surrounding for sensing gases. But, at times pristine graphene exhibits physisorption for the common gases and to increase the gas sensing ability one needs to introduce defect or functionalization to the pristine surface, which constrain the practicality to some extent. Moreover, to have a large surface area growth of graphene is a bottleneck as far as the industrial production is concerned. This opens up to the exfoliation of other 2D materials of the same group IV and thus silicene and germanene have been synthesized recently. Silicene has been successfully synthesized on Ag, Ir and ZrB2 substrate 9, 13-15, 17, whereas germanene has been grown on Pt (111) surface. There is also a successful experimental synthesis reporting the multilayer germanene that has been exfoliated on $\mathrm{SiO} 2 / \mathrm{Si}$ surface ${ }^{16-18}$. Similar to graphene, both silicene and germanene show some exciting properties like quantum hall effect ${ }^{19,20}$ and high carrier mobility ${ }^{20}$. In both silicene and germanene, the binding energies of common pollutants are more than that for graphene, which is a consequence of mixed $s p^{2}-s p^{3}$ hybridization. ${ }^{21}$ This also leads to the buckled structures in both the cases. Because of the buckled honeycomb structures, both silicene and germanene show stronger surface reactivity as compared to graphene and therefore chemisorption of the gas molecules is observed leading to the surface sensitivity.

In this theoretical investigation, we are exploring the sensing sensitivity of germanene nano sheets (Ge-NS) towards Hydrogen sulfide $\left(\mathrm{H}_{2} \mathrm{~S}\right)$ and Sulfur dioxide $\left(\mathrm{SO}_{2}\right)$ gas. One can find toxic $\mathrm{H}_{2} \mathrm{~S}$ in the fuel cell technology and catalyst poisoning can be overcome by detecting the presence of this gas. Our respiratory system can be affected due to the presence $\mathrm{SO}_{2}$ gas through inhalation and therefore we need a reasonable sensing material for this gas 
as well in our environment. So far, there have been no investigations reported for enhanced sensing ability towards $\mathrm{H}_{2} \mathrm{~S}$ and $\mathrm{SO}_{2}$ gases using $2 \mathrm{D}$ Ge-NS, as we believe that this material can be the promising choice for the practical sensing purpose.

We have envisaged different possibilities to enhance the sensing ability of Ge-NS towards $\mathrm{H}_{2} \mathrm{~S}$ and $\mathrm{SO}_{2}$. We have created mono, di and tri-vacancy in the Ge-NS as well as substituting the surface with different dopants like Fe, Co, Ni, Cu, Zn, As, Ga and Se. This wide range of substitution and defect possibilities would undergo the electronic structure calculations of Ge-NS in the presence of $\mathrm{H}_{2} \mathrm{~S}$ and $\mathrm{SO}_{2}$. Our theoretical calculations certainly guide the experimental investigation of Ge-NS with a preconceived idea about the suitable cases ideal for sensing $\mathrm{H}_{2} \mathrm{~S}$ and $\mathrm{SO}_{2}$ gas. We have also confirmed the stability of $2 \mathrm{D}$ Ge-NS layer induced with mono, di ad tri-vacancy and different functionalization. The sensing ability of Ge-NS for $\mathrm{H}_{2} \mathrm{~S}$ and $\mathrm{SO}_{2}$ will be determined from the variation in the electronic properties and the corresponding adsorption energy of all the considered cases. Our work shows that defected and substituted Ge-NS can certainly increase the surface-adsorbate interaction and as a consequence enhance the sensitivity for germanene based sensor devices.

\section{Computational details:}

The performed first principles calculations in this work are based on density functional theory and implemented by VASP code ${ }^{22,23}$. To keep an account of the ion-electron interactions, projector-augmented wave (PAW) approach is employed with an energy cut-off of $500 \mathrm{eV}^{24}$. We have used generalized gradient approximation (GGA) of Perdew-Burke-Ernzerhof type ${ }^{25}$ to consider exchange and correlation energies. Despite its widespread acceptability, GGA usually underestimates the binding energies, which are very important in case of weakly interacting systems especially the one under consideration. In order to have the more accurate adsorption mechanism we have included the dispersion term by adopting DFT-D2 scheme of Grimme ${ }^{26}$. A $4 \times 4 \times 1$ supercell of germanene having 32 atoms is used in this study. We have used Monkhorst-pack scheme for the sampling of Brillouin zone (BZ) with $5 \times 5 \times 1$ mesh for structural relaxation and $9 \times 9 \times 1$ mesh for determining the density of states ${ }^{27}$ of the corresponding structure with the minimum energy configurations. A vacuum of $15 \AA$ is inserted along Z-direction to avoid possible interaction between the periodically repeating monolayers. The charge transfer has been investigated based on the Bader charge analysis. ${ }^{28}$ The pristine, defected and functionalized structures are fully optimized until they reached an energy and forces limits of $10^{-5} \mathrm{eV}$ and $0.01 \mathrm{eV} / \AA ̊$ respectively. 


\section{Result and discussion:}

\subsection{Crystal Structure and Energetics of $\mathrm{H}_{2} \mathrm{~S}$ and $\mathrm{SO}_{2}$ on Pristine and Defected Ge-NS}

Before discussing the binding characteristics of $\mathrm{H}_{2} \mathrm{~S}$ and $\mathrm{SO}_{2}$ gases on pristine, defected and functionalized Ge-NS, we would describe the corresponding structural properties briefly. In optimized configurations as shown in fig-1, the lattice constant, Ge-Ge bond length and GeGe-Ge bond angle of pristine Ge-NS have been found as 4.06, $2.44 \AA$ and $112.44^{0}$ respectively. These values agree well with the previous studies ${ }^{29-31}$ and thus validate the rationality of computational methodology used here.

a) P-germanene

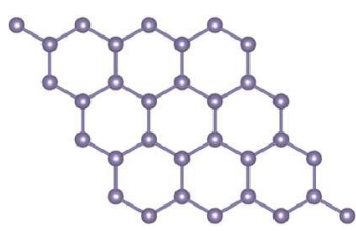

c) D-germanene

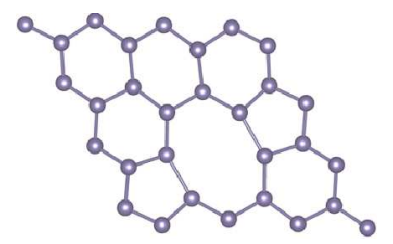

b) M-germanene

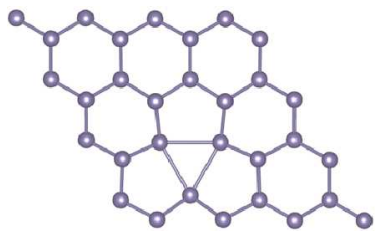

d) T-germanene

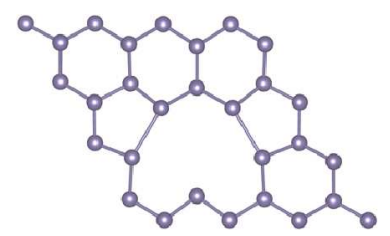

Fig-1. Optimized structures of (a) pure Ge-NS, (b) mono-vacancy Ge-NS, (c) di-vacancy GeNS, and (d) tri-vacancy Ge-NS. Blue spheres represent Ge atoms.

While considering the exposure of $\mathrm{H}_{2} \mathrm{~S}$ and $\mathrm{SO}_{2}$ on pristine Ge-NS, after exploring all the possible binding sites (bridge, hollow, top), and binding configurations of incident gases, H/S directed (for $\mathrm{H}_{2} \mathrm{~S}$ ) and $\mathrm{O} / \mathrm{S}$ directed (for $\mathrm{SO}_{2}$ ), we have observed relatively weak adsorption energies $\left(\mathrm{E}_{\mathrm{ads}}\right)$ of $-0.21 \mathrm{eV}$ for $\mathrm{H}_{2} \mathrm{~S}$ at a binding distance of $2.99 \AA$. However, a comparatively stronger adsorption ( $\mathrm{E}_{\mathrm{ads}}$ of $-0.64 \mathrm{eV}$ ) with lower surface-adsorbate distance of $2.09 \AA$ has been observed in case of $\mathrm{SO}_{2}$. The optimized structures of $\mathrm{H}_{2} \mathrm{~S}$ and $\mathrm{SO}_{2}$ adsorbed Ge-NS in their lowest energy configurations have been shown in Fig-2. One of the main characteristics of an effective nanosensors is to anchor the incident gases with $E_{a d s}$ that lies between physisorption and chemisorption, ideally close to $1 \mathrm{eV}^{31,32}$. Considering this criteria, Ge-NS do not serve the purpose of being reversible and efficient nanosensors in their pristine form, for sulfur containing gases due to inadequate binding. However, the binding mechanism of 
gas adsorption can be improved through defects formation and foreign element substitutions, as we will discuss both the phenomena below subsequently.

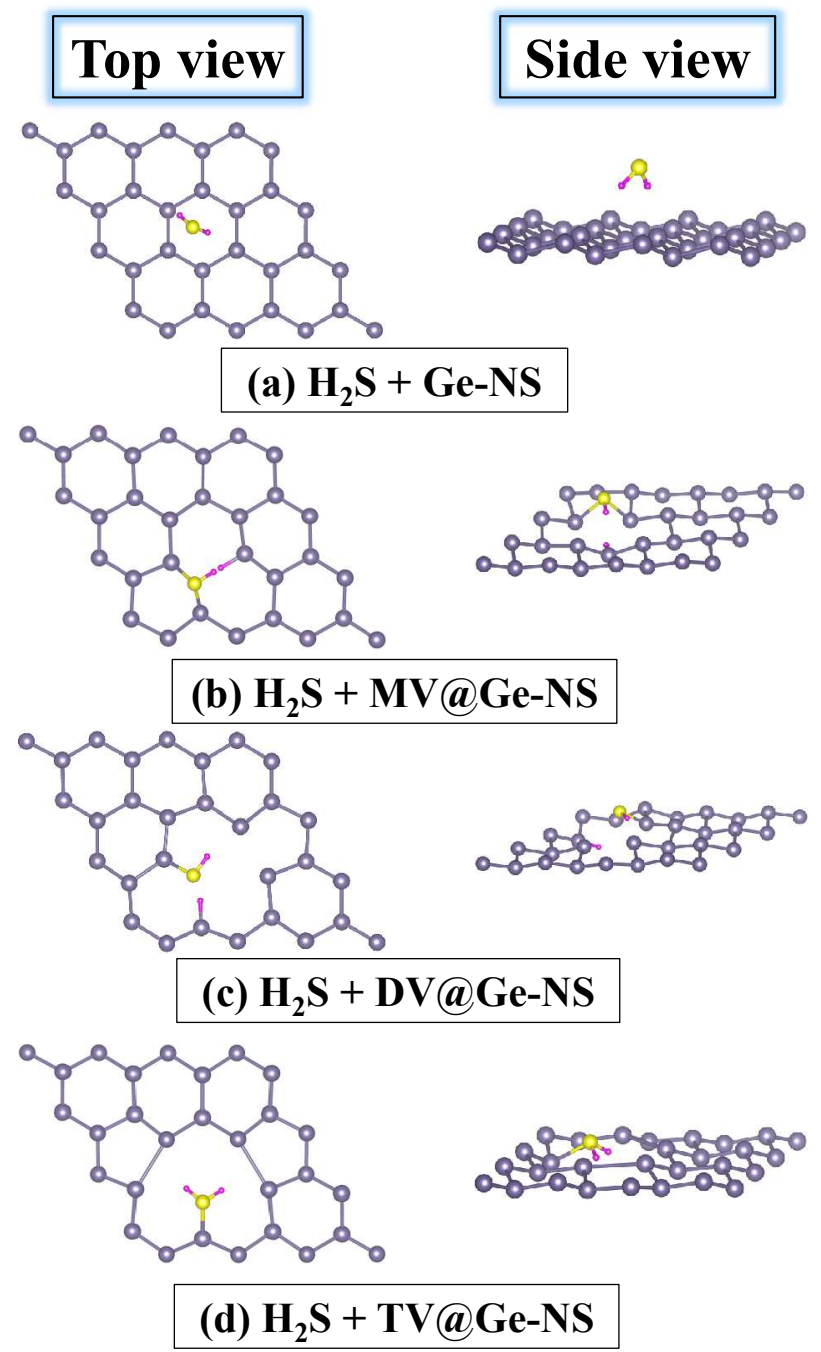


Fig-2. Optimized structures of $\mathrm{H}_{2} \mathrm{~S}$ molecule adsorbed on to germanene nanosheets (Ge-NS) with different kinds of defects (a) $\mathrm{H}_{2} \mathrm{~S}+\mathrm{Ge}-\mathrm{NS}$, (b) $\mathrm{H}_{2} \mathrm{~S}+\mathrm{MV} @ G e-\mathrm{NS}$, (c) $\mathrm{H}_{2} \mathrm{~S}+\mathrm{DV} @ \mathrm{Ge}-$ NS, and (d) $\mathrm{H}_{2} \mathrm{~S}+\mathrm{TV} @$ Ge-NS. Yellow and pink spheres represent sulfur and hydrogen atoms, respectively.

Defects are inescapable during the synthesis process of nanostructures; especially nano sheets and Ge-NS are no exceptions. We have restricted ourselves here to the vacancy defects created by removing single, double and triple Ge atoms from Ge-NS supercell, which are corresponding to $3.12 \%, 6.25 \%$ and $9.37 \%$ defects concentrations respectively. In case of mono vacancy $(\mathrm{MV})$, the structural properties changes upon ionic relaxations with the elongation of Ge-Ge bond length to $2.49 \AA$ and reduction of Ge-Ge-Ge bond angle to $110.2^{0}$. However, MV@Ge-NS is preserved its planer symmetry even under the exposure of $\mathrm{H}_{2} \mathrm{~S}$ and $\mathrm{SO}_{2}$, which bind differently to the defected Ge-NS. Here $\mathrm{H}_{2} \mathrm{~S}$ is binding in a dissociative (HS and $\mathrm{H}$ ) manner, such a way that one of its $\mathrm{H}$ atoms binds with the Ge atoms with a bond length of $1.58 \AA$ where the HS remains at a distance of $2.49 \AA$ above the sheet as shown in figure 2. The $\mathrm{E}_{\mathrm{ads}}$ of $\mathrm{H}_{2} \mathrm{~S}$ on MV@Ge-NS increases substantially with a value of around -1.35 $\mathrm{eV}$, which is 6.5 times greater than its value on pristine Ge-NS. The case of $\mathrm{SO}_{2}$ is different as compared to $\mathrm{H}_{2} \mathrm{~S}$, as the former preserves it molecular configuration upon its binding to MV@Ge-NS under the lowest energy configuration as shown in figure 2. However slight structural rearrangement is occurred in the relaxed $\mathrm{SO}_{2}$ structure with the elongation of one of the O-S bond lengths to $1.57 \AA$ and the other to $1.47 \AA$., while the O-S-O bond angle contracts to $109.43^{0}$ from $119^{\circ}$. The adsorption energy for $\mathrm{SO}_{2}$ on MV induced Ge-NS is $1.73 \mathrm{eV}$, which is higher as compared to its values on pristine Ge-NS and the corresponding surface-adsorbate distance is $1.99 \AA$. 


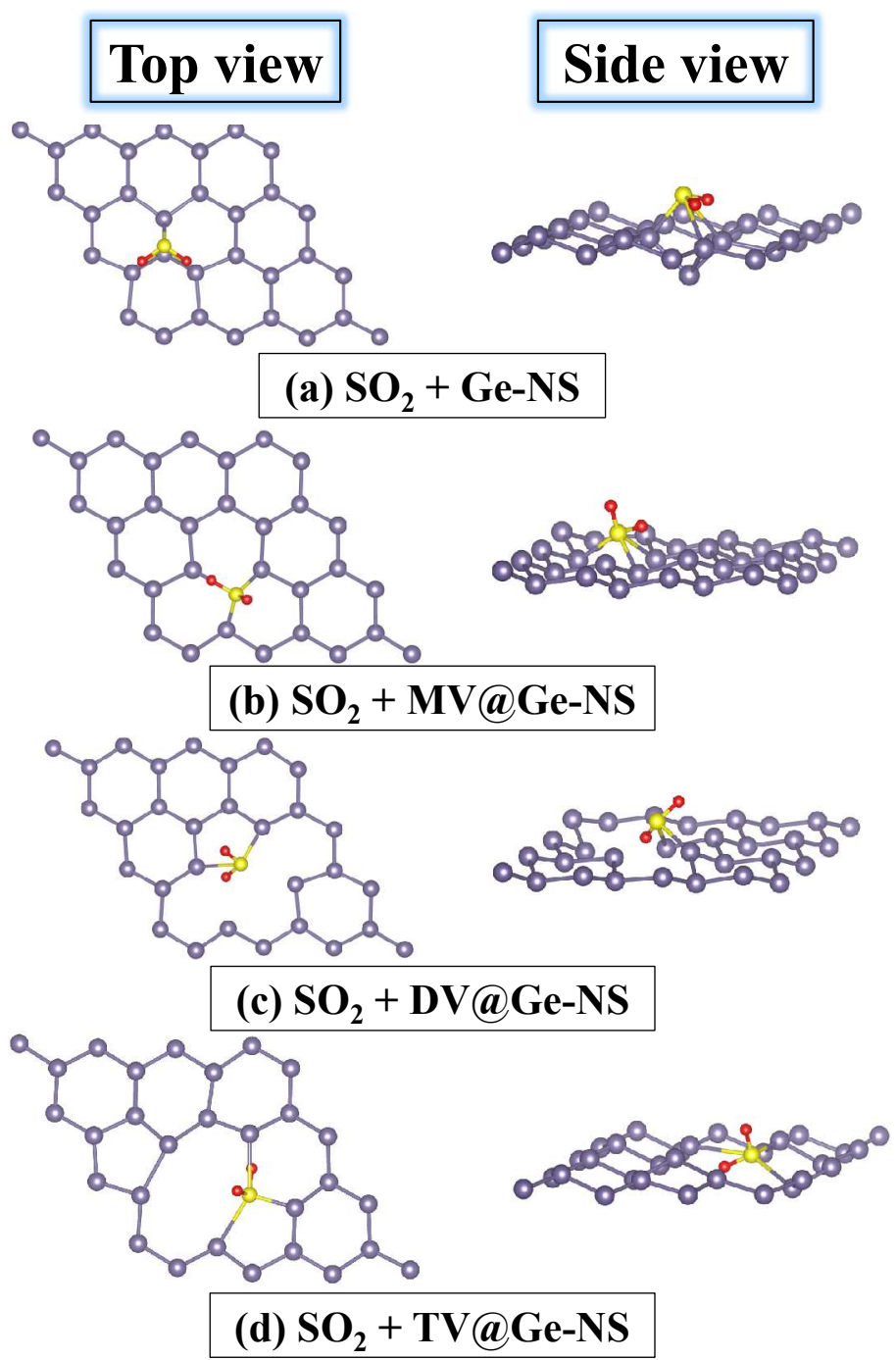

Fig-3. Optimized structures of $\mathrm{SO}_{2}$ molecule adsorbed on to germanene nanosheets (Ge-NS) with different kinds of defects (a) $\mathrm{SO}_{2}+\mathrm{Ge}-\mathrm{NS}$, (b) $\mathrm{SO}_{2}+\mathrm{MV} @ \mathrm{Ge}-\mathrm{NS}$, (c) SO $\mathrm{SO}_{2}$ DV@GeNS, and (d) $\mathrm{SO}_{2}+\mathrm{TV} @$ Ge-NS. Yellow and red spheres represent sulfur and oxygen atoms, respectively.

In case of divacancy (DV) of Ge-NS, the formation energy has been evaluated as 4.99 $\mathrm{eV}$, with the presence of two Ge-Ge bonds (2.58 $\AA$ and $2.94 \AA)$ in its lowest energy configurations. The introduction of $\mathrm{H}_{2} \mathrm{~S}$ at various doping sites has tuned out to the $\mathrm{H}_{2} \mathrm{~S}$ dissociation into H and HS similar to the case of MV@Ge-NS as shown in Fig-2. The dissociated $\mathrm{H}$ atom of $\mathrm{H}_{2} \mathrm{~S}$ binds to Ge with a bond length of $1.57 \AA$, whereas HS stays at $2.26 \AA$ above the DV@Ge-NS, with the resultant $\mathrm{E}_{\text {ads }}$ of $\mathrm{H}_{2} \mathrm{~S}$ in this case is $-1.12 \mathrm{eV}$.

In case of $\mathrm{SO}_{2}$ binding to DV@Ge-Ns, the lowest energy configuration corresponds to $\mathrm{E}_{\text {ads }}$ of 
$-0.93 \mathrm{eV}$ and a binding distance of $2.0 \AA$. Similar to the case of MV@Ge-NS, an elongation in O-S bond lengths ( $1.58 \AA$ and $1.49 \AA$ ) and reduction in O-S-O bond angle to $110.17^{0}$ have been observed in the relaxed structure of $\mathrm{SO}_{2}$ adsorbed DV@Ge-NS.

The formation energy for the case tri vacancy TV@Ge-NS is found to be $4.80 \mathrm{eV}$, whereas the Ge-Ge bond length and Ge-Ge-Ge bond angle are becoming $2.54 \AA$ and $113.6^{\circ}$ respectively in the relaxed configuration. Despite having small structural variations, TV@GeNS is preserving its planer geometry similar to MV@Ge-NS and DV@Ge-NS. In case of $\mathrm{H}_{2} \mathrm{~S}$ binding, unlike the previous two cases (MV@Ge-Ns, DV@Ge-NS), it binds to TV@GeNS in a way so that its molecular configuration preserves with adsorption energy and surface adsorbate distance are $-0.90 \mathrm{eV}$ and $2.53 \AA$ respectively. Small structural variations are occurred in $\mathrm{H}_{2} \mathrm{~S}$ upon relaxation of the gas molecule on TV@Ge-NS with elongation of H-S bond length from $1.34 \AA$ to $1.37 \AA$ and H-S-H bond angle from $92.1^{0}$ to $94.8^{0}$. Although $\mathrm{H}_{2} \mathrm{~S}$ binds relatively weakly on TV@Ge-NS as compared to MV@Ge-NS and DV@Ge-NS, however the resultant $E_{\text {ads }}$ is still 4.3 times higher than on pristine Ge-NS, which indicates that all the three defected systems are promising nanosensors for $\mathrm{H}_{2} \mathrm{~S}$ capture. In case of $\mathrm{SO}_{2}$ interaction with TV@Ge-NS, we have found a strong $\mathrm{E}_{\text {ads }}$ of $-1.49 \mathrm{eV}$ at a binding distance of $1.99 \AA$ A. Similar to its binding on MV@Ge-NS and DV@Ge-NS, $\mathrm{SO}_{2}$ molecule is rearranging itself upon structural relaxation on TV@Ge-NS with the elongation of one of its O-S bonds to $1.58 \AA$ and the other to $1.48 \AA$ from its ground state value of $1.43 \AA$. The O-S-O bond angle reduces from $119^{\circ}$ to $111.03^{\circ}$. The optimized structures of $\mathrm{H}_{2} \mathrm{~S}$ and $\mathrm{SO}_{2}$ on TV@GeNS are shown in in Figure 2 and 3, respectively.

\subsection{Crystal Structure and Energetics of $\mathrm{H}_{2} \mathrm{~S}$ and $\mathrm{SO}_{2}$ on Functionalized Ge-NS}

In this section, we would describe the promise of selected transition metals (TMs) substitution in Ge-NS to improve the sensing mechanism of $\mathrm{H}_{2} \mathrm{~S}$ and $\mathrm{SO}_{2}$. We have considered a wide range of TMs substituents, which includes $\mathrm{Fe}, \mathrm{Co}, \mathrm{Cu}, \mathrm{Ni}, \mathrm{Zn}, \mathrm{Ga}, \mathrm{Ge}$ and As. These types of substitutional doping have already been envisaged both experimentally and theoretically for other applications. ${ }^{33-36}$ The selection of substituents is based on their practicability to form uniform distribution with Ge-NS and therefore we have selected the elements showing low formations energies with Ge-NS. A single TM element replaces a $\mathrm{Ge}$ atom in Ge-NS corresponds to a lower substitutional doping of $3.125 \%$. The functionalization with $\mathrm{Fe}, \mathrm{Co}, \mathrm{Cu}$ and $\mathrm{Ni}$ are the cases, where improved binding of incident gases have been observed substantially. Thus, we would be explaining the structural, electronic and binding characteristics of these elements only. 


\section{Top view}

\section{Side view}
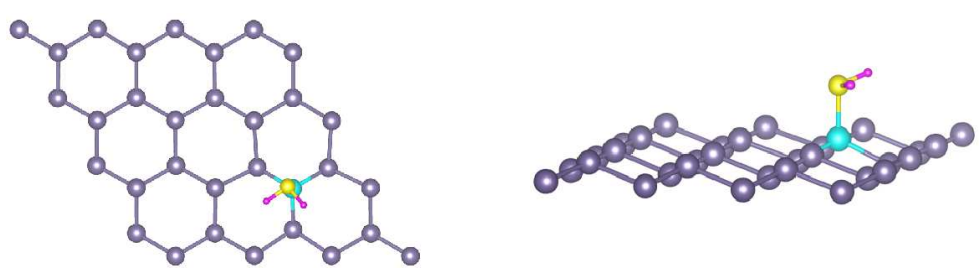

(a) $\mathrm{H}_{2} \mathrm{~S}+\mathrm{Fe} @ \mathrm{Ge}-\mathrm{NS}$
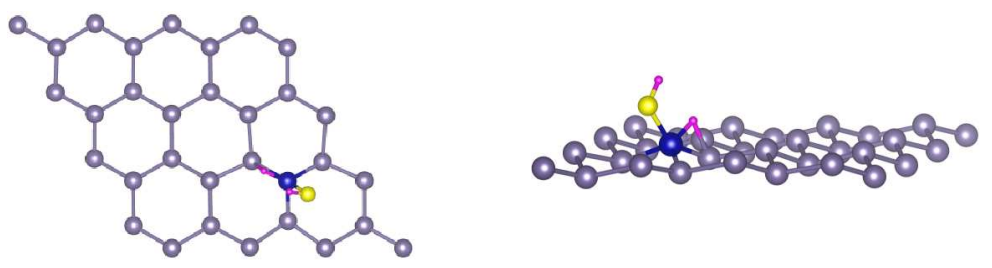

(b) $\mathrm{H}_{2} \mathrm{~S}+\mathrm{Co} @ G e-N S$
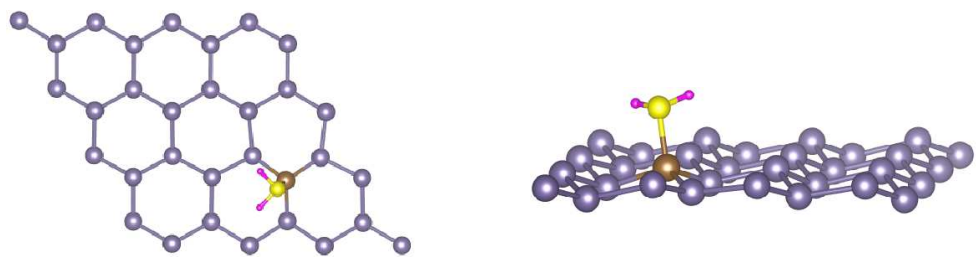

(c) $\mathrm{H}_{2} \mathrm{~S}+\mathrm{Ni} @$ Ge-NS
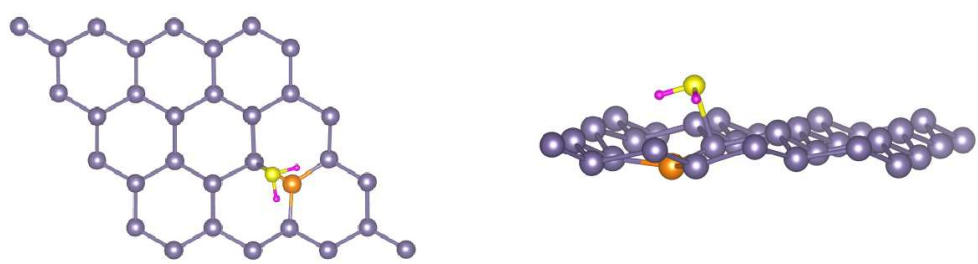

\section{(d) $\mathrm{H}_{2} \mathrm{~S}+\mathrm{Cu} @ \mathrm{Ge}-\mathrm{NS}$}

Fig-4. Optimized structures of $\mathrm{H}_{2} \mathrm{~S}$ molecule adsorbed on to the substituted germanene nanosheets with different types of substitutional doping (a) $\mathrm{H}_{2} \mathrm{~S}+\mathrm{Fe} @$ Ge-NS, (b) $\mathrm{H}_{2} \mathrm{~S}+$ Co@Ge-NS, (c) $\mathrm{H}_{2} \mathrm{~S}+\mathrm{Ni} @$ Ge-NS, and (d) $\mathrm{H}_{2} \mathrm{~S}+\mathrm{Cu} @$ Ge-NS. Yellow and pink spheres represent sulfur and hydrogen atoms, respectively.

The Fe substitution in Ge-NS is resulting (Fe@Ge-NS) into an exothermic reaction with formation energy of $-1.58 \mathrm{eV}$. In the minimum energy configuration, the Fe@Ge-NS corresponds to a relatively smaller Ge-Fe bond length of $2.27 \AA$ and Ge-Fe-Ge bond angle of 105. $7^{0}$. The binding of $\mathrm{H}_{2} \mathrm{~S}$ on $\mathrm{Fe} @ \mathrm{Ge}-\mathrm{NS}$ is occurred in such a way that the former preserves its molecular configurations without any substantial structural rearrangements and 
significantly high $\mathrm{E}_{\mathrm{ads}}$ of $-1.58 \mathrm{eV}$, which is almost 7.6 times larger than on the case of pristine Ge-NS. A minimum binding distance of $2.15 \AA$ is observed between Fe@Ge-NS and $\mathrm{H}_{2} \mathrm{~S}$. The $\mathrm{SO}_{2}$ binds even more strongly to $\mathrm{Fe} @ \mathrm{Ge}-\mathrm{NS}$ with $\mathrm{E}_{\mathrm{ads}}$ of $-1.84 \mathrm{eV}$ at a binding distance of $2.02 \AA$. Upon optimization, a small structural variation is occurred in $\mathrm{SO}_{2}$ with an increment of S-O bond to $1.46 \AA$ and reduction of O-S-O angle to $116.83^{\circ}$. The optimized structures of $\mathrm{Fe} @$ Ge-NS capturing $\mathrm{H}_{2} \mathrm{~S}$ and $\mathrm{SO}_{2}$ are shown in figure 4 and 5, respectively.

Similar to the case of Fe, Co substitution forming Co@Ge-NS is also an exothermic reaction with formation energy, Ge-Co bond length and Ge-Co-Ge bond angle are attaining the values of $-1.64 \mathrm{eV}, 2.29 \AA$ and $107.11^{0}$ respectively. Upon structural relaxation, the $\mathrm{H}_{2} \mathrm{~S}$ molecule is found to be dissociated into H and HS, while introduced to Co@Ge-NS resulting into a stronger binding of $-1.75 \mathrm{eV}$. The $\mathrm{Co}-\mathrm{H}$ and $\mathrm{Co}-\mathrm{HS}$ distances are found to be $1.60 \AA$ and $2.12 \AA$ respectively. In case of $\mathrm{SO}_{2}$ binding to $\mathrm{Co} @$ Ge-NS, similar adsorption energy ($1.75 \mathrm{eV}$ ) has been observed at a binding distance of $2.0 \AA$, however $\mathrm{SO}_{2}$ preserves its molecular symmetry without substantial structural changes in its lowest energy configuration. The optimized structures presented in Figure 4 and 5 show a significant increment of 8.4 and 2.71 times in the adsorption energies for both $\mathrm{H}_{2} \mathrm{~S}$ and $\mathrm{SO}_{2}$ respectively as compared to pristine Ge-NS.

The substitution of Ni into Ge-NS is resulted into Ni@Ge-NS with formation energy of $-0.54 \mathrm{eV}$, which is much lower than those of Fe and Co substitution. The Ni@Ge-NS has been relaxed with the associated Ge-Ni bond length of $2.30 \AA$ and Ge-Ni-Ge bond angle of $110.7^{0}$. The $\mathrm{H}_{2} \mathrm{~S}$ molecule binds with Ni@Ge-NS with $\mathrm{E}_{\mathrm{ads}}$ of $-1.20 \mathrm{eV}$ at a binding distance of $2.17 \AA$ preserving its molecular configuration. However, the H-S bond length of $\mathrm{H}_{2} \mathrm{~S}$ increases a little (1.336 $\AA$ in free state) to $1.364 \AA$ and H-S-H bond angle $\left(92.1^{0}\right.$ in free state) to $92.5^{0}$ upon adsorbing on Ni@Ge-NS. The binding of $\mathrm{SO}_{2}$ takes place at a binding distance of $2.05 \AA$ with the $-1.40 \mathrm{eV}$ as the adsorption energy. Even the $\mathrm{SO}_{2}$ molecule undergoes small structural alterations with $1.46 \AA$ as O-S bond length $\left(1.43 \AA\right.$ in free state) and $117.4^{0}$ O-S-O bond angle $\left(119^{0}\right.$ in free state). 


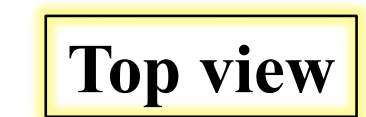

\section{Side view}

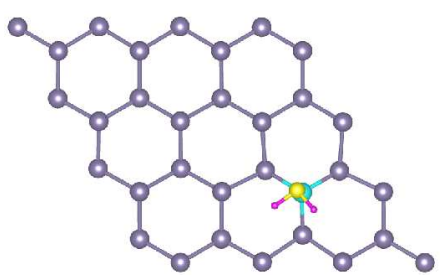

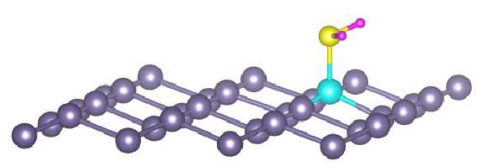

(a) $\mathrm{SO}_{2}+\mathrm{Fe} @ \mathrm{Ge}-\mathrm{NS}$

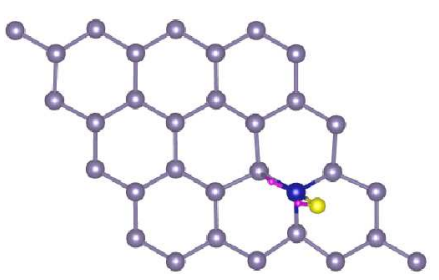

(b) $\mathrm{SO}_{2}+\mathrm{Co} @ G e-N S$

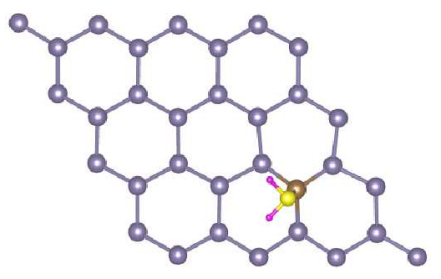

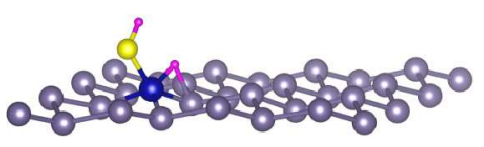

(c) $\mathrm{SO}_{2}+\mathrm{Ni} @ \mathrm{Ge}-\mathrm{NS}$

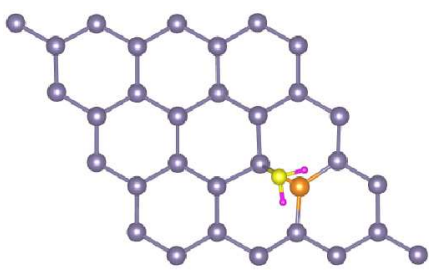

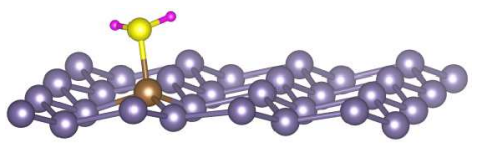

\section{(d) $\mathrm{SO}_{2}+\mathrm{Cu} @ \mathrm{Ge}-\mathrm{NS}$}

Fig-5. Optimized structures of $\mathrm{SO}_{2}$ molecule adsorbed on to the substituted germanene nanosheets with different types of substitutional doping (a) $\mathrm{SO}_{2}+\mathrm{Fe} @ \mathrm{Ge}-\mathrm{NS}$, (b) $\mathrm{SO}_{2}+$ Co@Ge-NS, (c) SO $\mathrm{SO}_{2}+\mathrm{Ni} @ G e-N S$, and (d) $\mathrm{SO}_{2}+\mathrm{Cu} @$ Ge-NS. Yellow, pink, cyan, blue, brown, and orange spheres represent sulfur, oxygen, iron, cobalt, nickel, and copper atoms, respectively.

Finally, $\mathrm{Cu}$ has been substituted in Ge-NS constituting $\mathrm{Cu} @$ Ge-NS with the formation energy of $-0.24 \mathrm{eV}$, which is the lowest among all the functionalization that are considered in this investigation. Here the optimized structure of $\mathrm{Cu} @ \mathrm{Ge}-\mathrm{NS}$ is having $\mathrm{Cu}-\mathrm{Ge}$ 
bond length of $2.34 \AA$ and Ge-Cu-Ge bond angle as $120.1^{\circ}$. Unlike the cases of Fe, Ni and Co substitution, the $\mathrm{Cu} @$ Ge-NS binds $\mathrm{H}_{2} \mathrm{~S}$ in relatively weak manner with $\mathrm{E}_{\text {ads }}$ of $-0.683 \mathrm{eV}$, however it is still 3.3 times higher than its value on pristine Ge-NS. The corresponding binding distance of $\mathrm{H}_{2} \mathrm{~S}$ from $\mathrm{Cu} @$ Ge-NS is $2.75 \AA$, which is also higher as compared to the other functionalization. Upon binding to $\mathrm{Cu} @ \mathrm{Ge}-\mathrm{NS}$, the $\mathrm{H}_{2} \mathrm{~S}$ remains in molecular form without being dissociating and substantial structural changes. Although $\mathrm{Cu}$ substitution improves the binding mechanism of $\mathrm{H}_{2} \mathrm{~S}$, however there is very small improvement on $\mathrm{SO}_{2}$ binding with $\mathrm{E}_{\text {ads }}$ of $-0.664 \mathrm{eV}$, a marginally higher than that of pristine Ge-NS case. The binding distance of $\mathrm{SO}_{2}$ to $\mathrm{Cu} @$ Ge-NS in ground state configuration is found to be $2.17 \AA$. The optimized structures of $\mathrm{H}_{2} \mathrm{~S}$ and $\mathrm{SO}_{2}$ are bonded with $\mathrm{Cu} @$ Ge-NS as depicted in Figure 4 and 5.

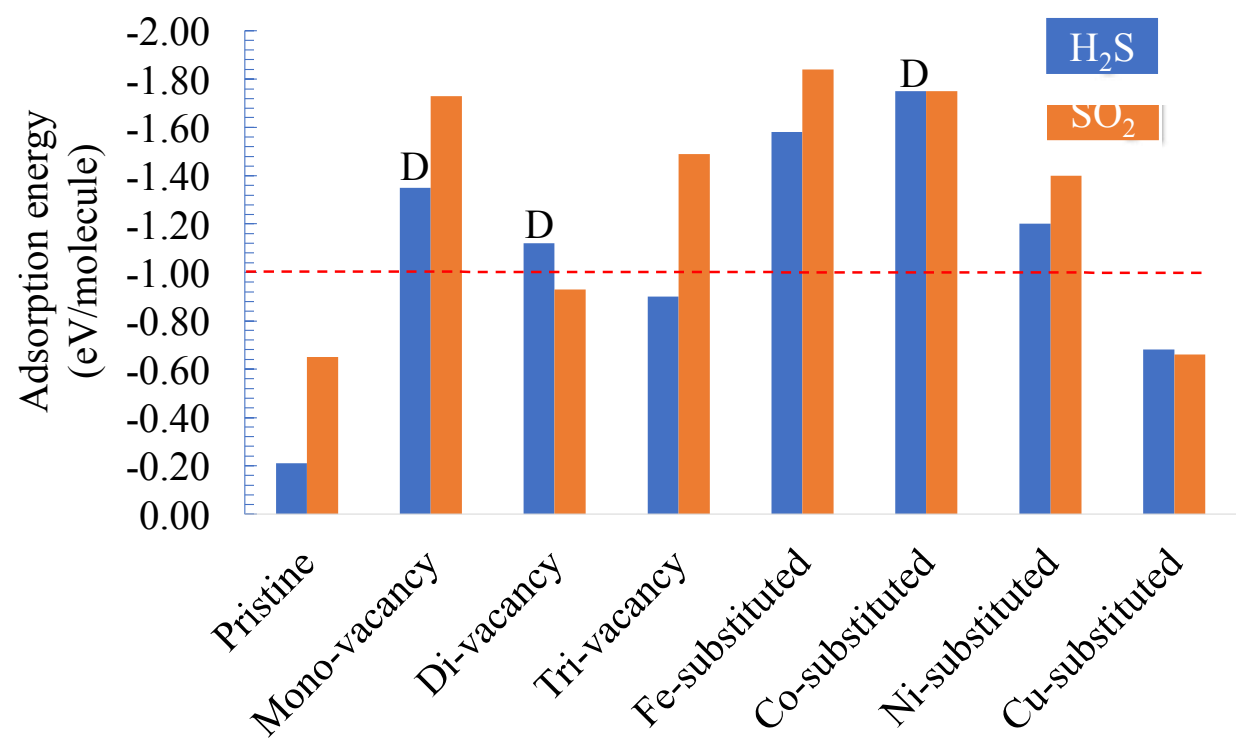

Fig-6. The calculated adsorption energies of $\mathrm{H}_{2} \mathrm{~S}$ and $\mathrm{SO}_{2}$ molecule adsorbed on to the various types of the germanene nanosheets. The dotted red line represents the probable adsorption energy that facilitates a reversible adsorption-desorption cycles with a reasonable recovery time by means of laser heating ${ }^{30,31}$ and $\mathrm{D}$ defines the dissociative adsorption.

\subsection{Density of States Analysis of Pristine, Defected and Functionalized Ge-NS under $\mathrm{H}_{2} \mathrm{~S}$ and $\mathrm{SO}_{2}$ exposure:}

The gas-detecting principles of chemical sensing devices rely on their measurable changes in the electrical conductivity induced by gas exposure ${ }^{37}$. The target gas molecules 
can either reduce or increase carrier concentrations via a charge transfer mechanism between the individual gas molecules and the sensing platform ${ }^{38}$. The transfer is basically triggered by the differences in electronegativity along with the chemical bonding, which could be reflected theoretically in the variation the adsorption energies for different systems. Hence, this parameter is one of the crucial descriptors for theoretically understanding the sensing mechanism. Figure 6 summarizes the adsorption energies of $\mathrm{H}_{2} \mathrm{~S}$ and $\mathrm{SO}_{2}$ adsorbed on different cases of germanene monolayer, that have been considered in this investigation. The ideal adsorption energy should be around $1.00 \mathrm{eV}$, which represents weak chemical interaction and it facilitates adsorption-desorption cycles with a reasonable recovery time by means of laser heating without the destruction of the corresponding surface structures ${ }^{31,32,39}$ of sensing materials. We have observed that, Di- and Tri-vacancy germanene are selectively suitable for detecting $\mathrm{SO}_{2}$ and $\mathrm{H}_{2} \mathrm{~S}$, respectively. On the other hand, surface functionalization by doping Ni offers an alternative way to enhance selectivity of germanene for both the Scontained gases. We will further focus on these systems by presenting the comprehensive analysis from the Density of States Analysis. 
Fig-7. Density of states (DOS) of (a) DV@Ge-NS, (b) SO $\mathrm{SO}_{2}$ DV@Ge-NS, (c) TV@Ge-NS, and (d) $\mathrm{H}_{2} \mathrm{~S}+\mathrm{TV} @$ Ge-NS. The dotted line represents the total DOS, while the green and blue lines account for the projected DOS onto $\mathrm{SO}_{2}$ and $\mathrm{H}_{2} \mathrm{~S}$ molecule, respectively. The Fermi level is positioned at 0 .

Figure 7(a-b) depict the corresponding DOS of DV@Ge-NS and $\mathrm{SO}_{2}+$ DV@Ge-NS. The presence of di-vacancy in germanene still preserves the metallic feature with the localized defect states right above the Fermi level. These states result in the disappearance of Dirac cone and the reduction in the transport property ${ }^{40}$. The $\mathrm{SO}_{2}$ adsorption leads to a substantial overlap between S- $3 p$ and Ge- $4 p$ orbitals in the range from -1.0 to $0.0 \mathrm{eV}$ (bonding) and from 1.0 to $1.5 \mathrm{eV}$ (anti-bonding), which is yielding a S-Ge covalent bond. In addition, there is one oxygen atom pointing towards DV@Ge-NS on which this oxygen tends to be accommodated at the missing germanium atom. As a result, the covalent $\mathrm{O}-\mathrm{Ge}$ bond is formed, which is described by the remarkable orbital overlap at -3.0 (bonding) to -4.0 (antibonding) $\mathrm{eV}$. Therefore, the formation of S-Ge and O-Ge covalent bonds influence the chemically strong adsorption energy between the $\mathrm{SO}_{2}$ molecule and DV@Ge-NS.

Similarly, the tri-vacancy yields loss of Dirac cone, as shown in figure 7(c-d), where the localized states at the Fermi level are observed. The adsorption of a $\mathrm{H}_{2} \mathrm{~S}$ molecule creates a covalent S-Ge bond, where the sulfur atom moves to the position of the missing germanium atom. This bond is originated from the overlap of S-3p and Ge- $4 p$ orbitals from the region of 
-4.0 to $-3.0 \mathrm{eV}$ and $1.0-2.0 \mathrm{eV}$. The enhanced adsorption energy of a $\mathrm{H}_{2} \mathrm{~S}$ molecule adsorbed on the TV@Ge-NS is originated from the presence of S-Ge bond. It is worth to mention here that, the presence of di- and tri-vacancy do not initiate magnetic moments most likely because of the buckling geometry leading to the stable non-magnetic singlet state ${ }^{42}$.

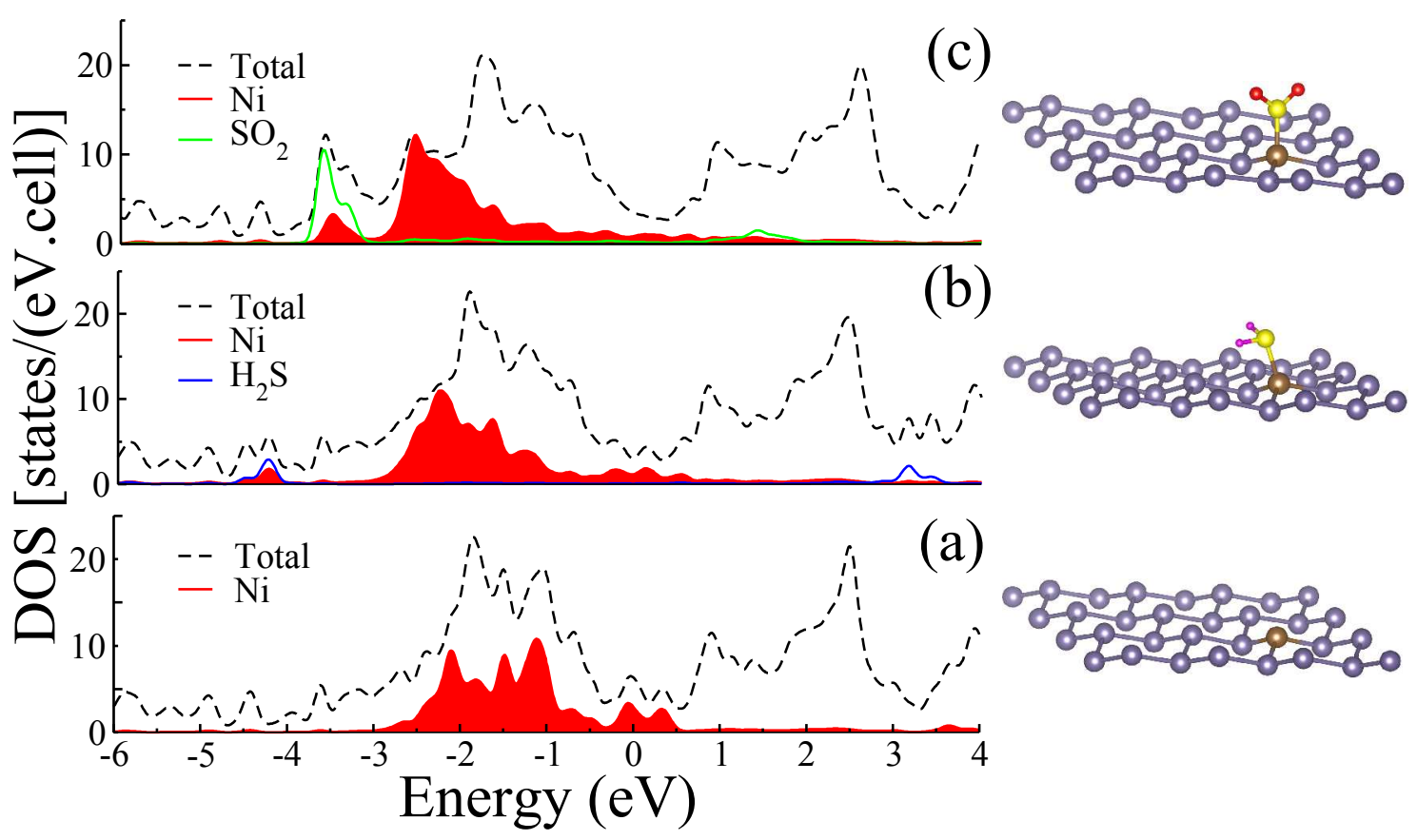

Fig-8. Density of states (DOS) of (a) Ni@Ge-NS, (b) $\mathrm{SO}_{2}+\mathrm{Ni}$ @Ge-NS, and (c) $\mathrm{H}_{2} \mathrm{~S}+$ Ni@Ge-NS. The dotted line represents the total DOS, while the green and blue lines account for the projected DOS onto $\mathrm{SO}_{2}$ and $\mathrm{H}_{2} \mathrm{~S}$ molecule, respectively. The Fermi level is positioned at 0 .

Figure 8 (a-d) show DOS of DV@Ge-NS, SO + DV@Ge-NS, TV@Ge-NS, and $\mathrm{H}_{2} \mathrm{~S}$ +TV@Ge-NS respectively. Ni-substituted germanene possesses the localized $3 d$ states, which predominantly overlap with Ge- $4 p$ states in the region between -3.0 to $0.5 \mathrm{eV}$. This overlap results in $d^{2} p$ hybridization with the trigonal pyramidal geometry and the coordination number of $3^{41}$. There is a non-bonded electron from Ni-4s as indicated by the peak of DOS at Fermi level. The adsorption of a $\mathrm{H}_{2} \mathrm{~S}$ molecule on the Ni-doped germanene creates a Ni-S bond as described by the appreciable overlap of S- $3 p$ and Ni-3d at around $-4.2 \mathrm{eV}$ (bonding) and $3.3 \mathrm{eV}$ (anti-bonding). Meanwhile, the adsorption of $\mathrm{SO}_{2}$ yields a Ni-S bond with a notable overlap at around $-3.5 \mathrm{eV}$ (bonding) and $1.5 \mathrm{eV}$ (anti-bonding). In both the cases of $\mathrm{H}_{2} \mathrm{~S}$ and $\mathrm{SO}_{2}$, the sulfur atom is acting as the bridging bond between the surface and the adsorbate. However, the orbital overlap of Ni-S bond occurs at the different energies. This is fundamentally because the overlap of $\mathrm{S}$ and $\mathrm{O}$ in $\mathrm{SO}_{2}$ is at the higher energy as compared to 
that of $\mathrm{S}$ and $\mathrm{H}$ in $\mathrm{H}_{2} \mathrm{~S}$. It should be mentioned that the orbital overlap between $\mathrm{S}$ and $\mathrm{Ni}$ consequently shifts the $d$-band to the lower energy as compared the previous case of adsorption. Furthermore, there are no magnetic moments in Ni-substituted germanene because of the fact that, Ni has $d^{8}$ character from which one vacant- $d$ band is used to construct $d^{2} p$ hybrid to later bind with three surrounding Ge atoms. Overall, it is concluded that the Ni-S bond play a deciding role in the strong adsorption energy for $\mathrm{H}_{2} \mathrm{~S}$ and $\mathrm{SO}_{2}$ molecules adsorbed on the $\mathrm{Ni}$-doped germanene.

\section{Conclusion:}

In a quest of designing an effective nanosensors based on large surface area of 2D monolayers, operating in the feasible operating conditions reversibly, we have envisaged the promise of Ge-NS. The sensing behavior of two of the most important sulfur containing gases, $\mathrm{H}_{2} \mathrm{~S}$ and $\mathrm{SO}_{2}$, on pristine, defected and transition metals substituted Ge-NS, have been studied by employing the first principles calculations based on DFT. Our energetic analysis based on van der Waals corrected simulations has suggested that the incident gases do not yield desirable binding on pristine Ge-NS. The introduction of vacancy defects by removing single; double and triple Ge atoms from a supercell constituting mono, di and tri vacancies, which improves the binding of both $\mathrm{H}_{2} \mathrm{~S}$ and $\mathrm{SO}_{2}$ to a great extent. In addition to vacancy defects, the substitution of Ge with a range of transition metals like As, $\mathrm{Co}, \mathrm{Cu}, \mathrm{Fe}, \mathrm{Ga}, \mathrm{Ge}$ $\mathrm{Ni}$ and $\mathrm{Zn}$ also enhance the sensing characteristics of both $\mathrm{H}_{2} \mathrm{~S}$ and $\mathrm{SO}_{2}$. Apart from the fact of improved binding, the apparent variations in the electronic properties calculated based on the total and projected density of states before and after the gas exposure also indicate the promise of defected and substituted Ge-NS as an efficient nanosensor for $\mathrm{H}_{2} \mathrm{~S}$ and $\mathrm{SO}_{2}$.

\section{Acknowledgments:}

The Nanotechnology Centre (NANOTEC), NSTDA Ministry of Science and Technology (Thailand) have supported TK and VA through its program of Centre of Excellence Network, Integrated Nanotechnology Research Centre Khon Kaen University (Thailand). TH is indebted to the resources at NCI National Facility systems at the Australian National University through National Computational Merit Allocation Scheme supported by the Australian Government and the University of Queensland Research Computing Centre. SC and RA acknowledge the Swedish Research Council (VR), Carl Tryggers Stiftelse för 
Vetenskaplig Forskning and StandUp for financial support. The SNIC and UPPMAX are also acknowledged for provided computing time.

\section{References:}

1. Hussain, T.; Hankel, M.; Searles, D. J. Improving Sensing of Sulfur-Containing Gas Molecules with ZnO Monolayers by Implanting Dopants and Defects. J. Phys. Chem. C 2017, 121, 24365-24375.

2. Tomchenko, A. A.; Harmer, G. P.; Marquis, B. T.; Allen, J. W. Semiconducting Metal Oxide Sensor Array For The Selective Detection of Combustion Gases. Sensor. Actuat B: Chem. 2003, 93, 126-134.

3. Arafat, M.; Dinan, B.; Akbar, S.A.; Haseeb, A. Gas Sensors Based on One Dimensional Nanostructured Metal-Oxides: A Review. Sensors, 2012, 12, 7207-7258.

4. Verghese, S. S.; Verghese, S. H.; Swaminathan, S.; Singh, K. K.; Mittal, K. TwoDimensional Materials for Sensing: Graphene and Beyond. Electronics, 2015, 4, 651687

5. Li, J.; Lu, Y.; Ye, Q.; Cinke, M.; Han, J.; Meyyappan, M. Carbon Nanotube Sensors for Gas and Organic Vapour Detection. Nano Lett. 2003, 3, 929-933.

6. Hussain, T.; Kaewmaraya, T.; Khan, M.; Chakraborty, S.; Islam, M. S.; Amornkitbamrung, V. Ahuja, R. Improved Sensing Characteristics of Methane over Zno Nano Sheets Upon Implanting Defects and Foreign Atoms Substitution. Nanotechnology, 2017, 28, 415502-415507.

7. Comini, E.; Sberveglieri, G. Metal Oxide Nanowires as Chemical Sensors. Mater. Today. 2010, 13, 36-44.

8. Cui, Y.; Wei, Q.; Park, H.; Lieber, C.M. Nanowire Nanosensors for Highly Sensitive and Selective Detection of Biological and Chemical Species. Science, 2001, 293, 1289-1292.

9. Chen, X.; Wong, C.K.Y.; Yuan, C.A.; Zhang, G. Nanowire-Based Gas Sensors. Sensor. Actuat B: Chem. 2013, 177, 178-195.

10. Liu, X.; Cheng, S.; Liu, H.; Hu, S.; Zhang, D.; Ning, H. A Survey on Gas Sensing Technology. Sensors, 2012, 12, 9635-9665.

11. Mahabal, M. S.; Deshpande, M. D. Hussain, T.; Ahuja, R. Sensing Characteristics of Phosphorene Monolayers toward $\mathrm{PH}_{3}$ and $\mathrm{Ash}_{3}$ Gases upon the Introduction of Vacancy 
Defects. J. Phys. Chem. C, 2016, 120, 20428-20436.

12. Huang, X. J.; Choi, Y. K. Chemical Sensors Based on Nanostructured Materials. Sensor. Actuat B: Chem. 2007, 122, 659-671.

13. Islam, M. S.; Hussain, T.; Rao, G. S.; Panigrahi, P.; Ahuja, R. Augmenting The Sensing Aptitude of Hydrogenated Graphene by Crafting With Defects and Dopants. Sensor. Actuat B: Chem. 2016, 228, 317-321.

14. Feng, B.; Ding, Z.; Meng, S.; Yao, Y.; He, X.; Cheng, P.; Chen, L.; Wu, K. Evidence of Silicene in Honeycomb Structures of Silicon On Ag(111). Nano Lett. 2012, 12, 3507-11.

15. Meng, L.; Wang, Y.; Zhang, L.; Du, S.; Wu, R.; Li, L.; Zhang, Y.; Li, G.; Zhou, H.; Hofer, W. A.; Gao, H. J. Buckled Silicene Formation On $\operatorname{Ir}(111)$.Nano Lett., 2013,13, 685-690.

16. Davila, M. E.; Lay, G. L. Few Layer Epitaxial Germanene: A Novel Two-Dimensional Dirac Material. Sci. Reports 2015, 6, 20714-20722.

17. Bianco, E.; Butler, S.; Jiang, S.; Restrepo, O. D.; Windl, W.; Goldberger, J. E. Stability and Exfoliation of Germanane: A Germanium Graphane Analogue. ACS Nano, 2013, 7, 4414-4421.

18. Ma, Y.; Dai, Y.; Niu, C.; Huang, B. Halogenated Two-Dimensional Germanium: Candidate Materials for Being of Quantum Spin Hall State. J. Mater. Chem. 2012, 22, 12587-12591.

19. Bechstedt, F.; Matthes, L.; Gori, P.; Pulci, O. Infrared Absorbance of Silicene and Germanene. Appl. Phys. Lett. 2012, 100, 261906-261908.

20. Kresse, G.; Hafner, J. Ab Initio Molecular Dynamics for Liquid Metals. Phys. Rev. B 1993, 47, 558-561.

21. Hussain, T.; Kaewmaraya, T.; Chakraborty, S.; Ahuja, R. J. Phys. Chem. C 2016, 120, 25256-25262.

22. Kresse, G.; Hafner, J. Ab Initio Molecular-Dynamics Simulation of the Liquid-MetalAmorphous-Semiconductor Transition in Germanium. Phys. Rev. B 1994, 49, 1425114269.

23. Blochl, P. E. Projector Augmented-Wave Method. Phys. Rev. B 1994, 50, 17953-17979.

24. Perdew, J. P.; Burke, K.; Ernzerhof, M. Generalized Gradient Approximation Made Simple. Phys. Rev. Lett. 1996, 77, 3865-3868.

25. Grimme, S. Semiempirical GGA-Type Density Functional Constructed With A LongRange Dispersion Correction. J. Comput. Chem., 2006, 27, 1787-1799.

26. Monkhorst, H. J.; Pack, J. D. Special Points for Brillouin-Zone Integrations. Phys. Rev. B 1976, 13, 5188-5192. 
27. Bader, R. F. W., Atoms in Molecules - A Quantum Theory; Oxford University Press: Oxford, 1990.

28. Zhu, L.; Chang, X.; He, D.; Xue, Q.; Li, X.; Jin, Y.; Zheng, H.; Ling, C. Defective Germanene As A High-Efficiency Helium Separation Membrane: A First Principles Study. Nanotechnology, 2017, 28, 135703-9.

29. Pang, Q.; Zhang, C.; Li, L.; Fu, Z.; Wei, X.; Song, Y. Adsorption of Alkali Metal Atoms on Germanene: A First-Principles Study. Appl. Sur. Sci. 2014, 314, 15-20.

30. Liu, G.; Liu, S. B.; Xu, B.; Ouyang, C. Y.; Song, H. Y. First-Principles Study of The Stability of Free-Standing Germanene in Oxygen Atmosphere. J. App. Phys. 2015, 118, 124303-124308.

31. Tawfik, S. A.; Cui, X. Y.; Carter, D. J.; Ringer, S. P.' Stampfl, C. Sensing SulfurContaining Gases Using Titanium and Tin Decorated Zigzag Graphene Nanoribbons From First-Principles. Phys. Chem. Chem. Phys. 2015, 17, 6925-6932.

32. Huang, B.; Li, Z.; Liu, Z.; Zhou, G.; Hao, S.; Wu, J.; Gu, B.; Duan, W. Adsorption of Gas Molecules on Graphene Nanoribbons and Its Implication for Nanoscale Molecule Sensor. J. Phys. Chem. C, 2008, 112, 13442-13446.

33. Robertson, A. W.; Montanari, B.; He, K.; Kim, J.; Allen, C. S.; Wu, Y. A.; Olivier, J.; Neethling, J.; Harrison, N.; Kirkland, A. I.; Warner, J. H. Dynamics of Single Fe Atoms in Graphene Vacancies. Nano Lett. 2013, 13, 1468-1475.

34. Chisholm, M. F.; Duscher, G.; Windl, W. Oxidation Resistance of Reactive Atoms in Graphene. Nano Lett. 2012, 12, 4551-4655.

35. Rodríguez-Manzo, J. A.; R.; Cretu, O.; Banhart, F. Trapping of Metal Atoms in Vacancies of Carbon Nanotubes and Graphene. ACS Nano, 2010, 4, 3422-3428.

36. Sun, M.; Ren, Q.; Zhao, Y.; Wang, S.; Yu, J.; Tang, W. Magnetism in Transition MetalSubstituted Germanane: A Search for Room Temperature Spintronic Devices. J. App. Phys. 2016, 119, 143904-143911.

37. Varghese, S. S.; Varghese, S. H.; Swaminathan, S.; Singh, K. K.; Mittal, V. TwoDimesnsional Materials for Sensing: Graphene and Beyond. Electronic, 2015, 4, 651-687

38. Schedin, F.; Geim, A. K.; Morozov, S. V.; Hill, E. W.; Blake, P.; Katsnelson, M. I.; Novoselov, K. S. Detection of Individual Gas Molecules Adsorbed on Graphene. Nat. Mater. 2007, 6, 652-655.

39. Zhang, Y.; Chen, Y.; Zhou, L.; Liu, C.; Zeng, J.; Zhang, H.; Peng, Y. Improving Gas 
Sensing Properties of Graphene by Introducing Dopants and Defects: A First-Principles Study. Nanotechnology, 2009, 20, 185504

40. Padilha, J. E.; Pontes, R. B. Electronic and Transport Properties of Structural Defects in Monolayer Germanene: An Ab Initio Investigation. Solid State Commun. 2016, 225, 3843.

41. Bard, A. J.; Faulker, L. R. Electrochemical Methods, 2nd ed.; Wiley: New York, 2001.

42. Ali, M.; Pi, X.; Liu, Y.; Yang, D. Electronic and Magnetic Properties of Graphene, Silicene and Germanene with Varying Vacancy Concentration. AIP Advances 2017, 7, 045308 . 


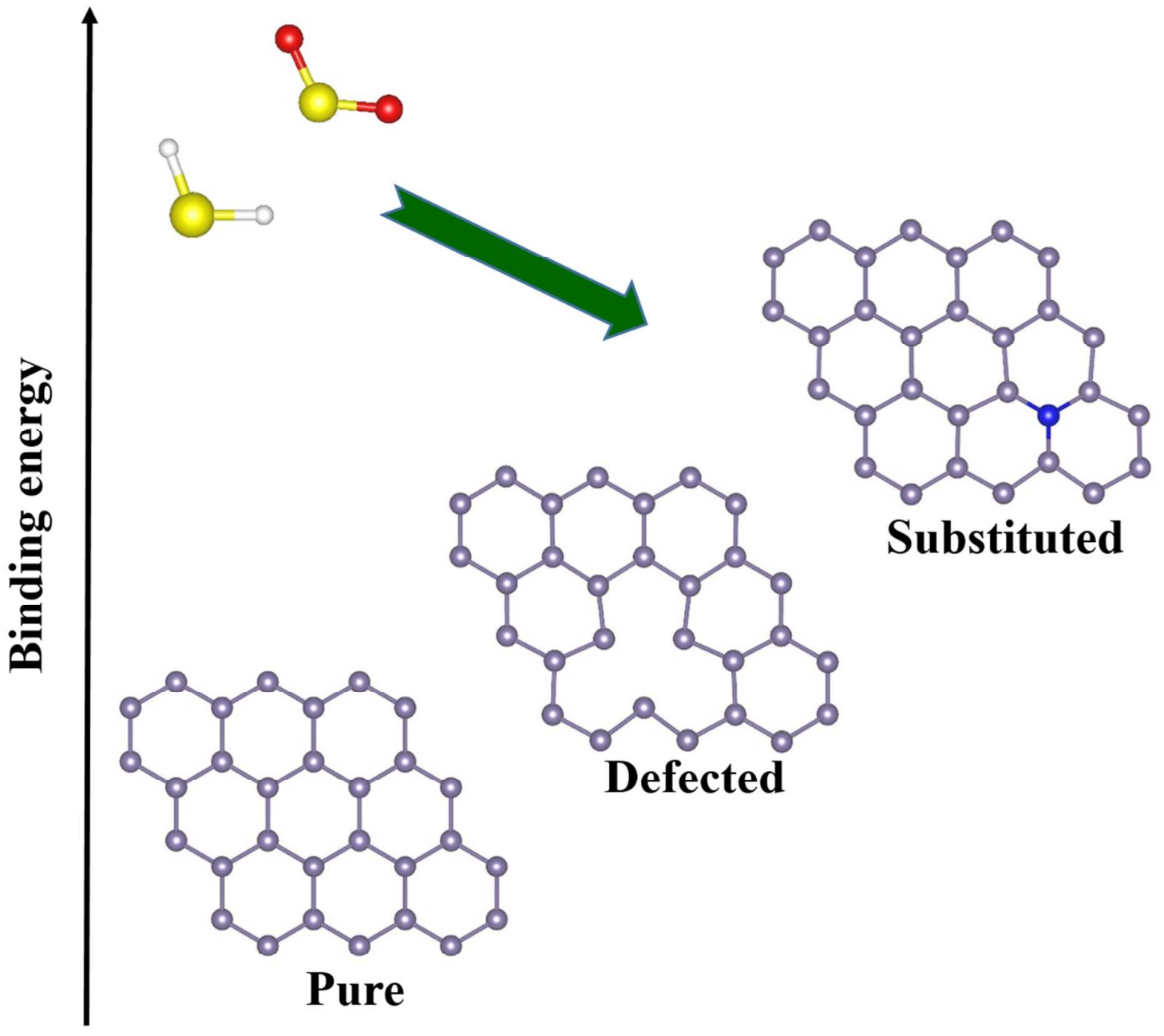

For TOC only 